Check for updates

Cite this: RSC Adv., 2019, 9, 28534

Received 9th June 2019

Accepted 4th September 2019

DOI: 10.1039/c9ra04321g

rsc.li/rsc-advances

\section{Facile synthetic approach towards vasorelaxant active 4-hydroxyquinazoline-4-carboxamides $\dagger$}

\author{
Marian N. Aziz, ${ }^{a}$ Siva S. Panda, (D) ${ }^{b}$ ElSayed M. Shalaby, (D) ${ }^{c}$ Nehmedo G. Fawzy ${ }^{a}$ \\ and Adel S. Girgis (iD *a
}

A Facile synthetic approach is reported towards 4-hydroxyquinazoline-4-carboxamides 13a-i through ring expansion of 2,3-dioxoindoline-1-carboxamides $10 a-c$ during secondary amine 11ad nucleophilic reaction. Single crystal X-ray studies of $10 \mathrm{c}$ and $13 \mathrm{~d}$ support the structures. Some of the synthesized quinazolinecarboxamides 13 show promising vasorelaxant properties with potency higher than that of Doxazosin through the pre-contracted (norepinephrine hydrochloride) rat aorta standard bioassay. Good molecular models (2D-QSAR, pharmacophore) describe the biological observations.

\section{Introduction}

Quinazoline is an important heterocyclic system that occupies a striking position in medicinal chemistry due to the diverse biological and/or pharmacological properties associated with its derivatives. ${ }^{1-6}$ For example, Gefitinib (Iressa) $\mathbf{1}$ is a drug clinically approved by the FDA for treatment of non-small cell lung cancer (NSCLC, on May 5, 2003) (Fig. 1). It is a tyrosine kinase inhibitor accessible for patients with metastatic NSCLC., ${ }^{7,8}$ Erlotinib (Tarceva) 2 is also a kinase inhibitor approved by the FDA (Nov. 18, 2004) for locally advanced or metastatic NSCLC and pancreatic cancers. ${ }^{9,10}$ Lapatinib (Tykerb) 3 is approved by the FDA (Mar. 13, 2007) for treatment of advanced or metastatic breast cancer. ${ }^{11,12}$ However, due to the drug resistance observed by the targeted first generation reversible EGFR (epidermal growth factor receptor) drugs 1-3, by many patients, the second generation irreversible EGFR inhibitors were developed. ${ }^{13}$ Of which, Afatinib (Gilotrif) 4 and Dacomitinib (Vizimpro) 5 were approved for treatment of metastatic NSCLC (FDA on July 12, 2013 and Sept. 27, 2018, respectively). ${ }^{14-17}$

Additionally, Doxazosin 6 and Terazosin 7 are well known drugs for hypertension. ${ }^{18}$ Hypertension is one of the cardiovascular diseases which are the first cause of human death

${ }^{a}$ Department of Pesticide Chemistry, National Research Centre, Dokki, Giza 12622, Egypt.E-mail: girgisas10@yahoo.com

${ }^{b}$ Department of Chemistry \& Physics, Augusta University, Augusta, GA 30912, USA

${ }^{c} X$-Ray Crystallography Lab, Physics Division, National Research Centre, Dokki, Giza 12622, Egypt

$\dagger$ Electronic supplementary information (ESI) available: Copy of IR, ${ }^{1} \mathrm{H}$ - and ${ }^{13} \mathrm{C}-\mathrm{NMR}$ spectra, biological curves, computational tables/figures. CCDC 1913146 and 1913147. For ESI and crystallographic data in CIF or other electronic format see DOI: $10.1039 /$ c9ra04321g globally (approximately 17.9 millions representing 31\% of global deaths in 2016 according to WHO "World Health Organization"19,20). Heart attack and stroke are usually associated with hypertension. ${ }^{21}$ Many $\alpha_{1}$-adrenergic receptor $\left(\alpha_{1}{ }^{-}\right.$ AR) antagonists are clinically useful drugs for vascular smooth muscle relaxation (vasodilator) such as Doxazosin 6 and Terazosin 7 but associate with severe side effects. ${ }^{22}$ The high mortality factor of hypertension and serious side effects of the clinically known drugs dimensioned their applications, directed the research efforts for developing novel effective hits/leads. The recent publications describing diverse biological properties of quinazoline containingcompounds as antibacterial, ${ }^{23}$ antiviral, ${ }^{24}$ antifungal, ${ }^{25}$ antiplasmodial, ${ }^{26}$ anti-inflammatory,,$^{27}$ cholinesterase, ${ }^{28,29}$ and monoamine oxidase inhibitors ${ }^{30}$ also prompted the present work.

The present study is directed towards construction of novel quinazoline-based analogues and investigation their vasorelaxant properties. The amino group located at the 4position of the antihypertensive active drugs 6 and 7 were replaced by a hydroxy group due to the common chemical properties of the two functions. The alicyclic-amino ring is also attached to the quinazolinyl C-4 forming an amidic linkage (Scheme 1). Many synthetic pathways were developed for construction of quinazoline-containing compounds including $m$-chloroperbenzoic acid oxidative rearrangement of 4-imino-(1H,4H)-3,1-benzoxazin-2-ones and reaction of isatins with arylamines in the presence of hydrogen peroxide as an oxidant. ${ }^{31}$ Previous publications described the ring opening of isatins under the effect of water, alcohols, amines, ${ }^{32,33}$ urea, $^{34}$ hydrazines ${ }^{33,35}$ and guanidine. ${ }^{34,36}$ The present study describes a facile synthetic approach via ring expansion of isatin-1-carboxamides during secondary amine nucleophilic attack. 
<smiles></smiles><smiles>COc1cc2ncnc(Nc3ccc(F)c(Cl)c3)c2cc1NC(=O)/C=C/CN1CCCCC1</smiles><smiles>COc1cc2nc(N3CCN(C(=O)C4COc5ccccc5O4)CC3)nc(N)c2cc1OC</smiles>

Doxazosin 6<smiles>COc1cc2nc(N3CCN(C(=O)C4CCCO4)CC3)nc(N)c2cc1OC</smiles>

Terazosin 7

Fig. 1 Quinazoline-based antitumor and antihypertensive drugs.

\section{Results and discussion}

\section{Chemistry}

Isocyanates $\mathbf{9 a , b}$ were subjected to reaction with isatins $\mathbf{8 a}, \mathbf{b}$ in dry tetrahydrofuran (THF) in the presence of sufficient amount of triethylamine at $0{ }^{\circ} \mathrm{C}$ affording the corresponding 2,3-dioxoindoline-1-carboxamides 10a-c in good yields which were used in the next reaction without any further purification. $N$-Ethyl-2,3-dioxoindoline-1-carboxamide (10c) could be isolated in good microcrystallized form accessible for single crystal X-ray studies, which add good support for the structure (Fig. 2).

Reaction of 10a-c with secondary amines 11a-d in dry THF led to ring expansion affording directly the 4hydroxyquinazoline-4-carboxamides $13 \mathbf{a}-\mathbf{i}$ and not the expected 3-hydroxy-2-oxoindoline-1-carboxamides 12 . IR<smiles>[R]c1ccc2c(c1)C(=O)C(=O)N2</smiles>

8<smiles>[R]NC(=O)N1C(=O)C(=O)c2cc([R])ccc21</smiles>

$H_{N R}^{2} R^{3}(11)$ THF

10

13a; $R=H, R^{1}=P h, N R^{2} R^{3}=$ Diethylamino $((71 \%$ yield $)$

13b; $R=H, R^{1}=P h, N R^{2} R^{3}=$ Pyrrolidinyl $(74 \%$ yield $)$

13c; $R=C l, R^{1}=P h, N R^{2} R^{3}=$ Pyrrolidinyl $(85 \%$ yield $)$

13d; $R=H, R^{1}=P h, N R^{2} R^{3}=$ Piperidinyl $(77 \%$ yield $)$

13e; $R=\mathrm{Cl}, \mathrm{R}^{1}=\mathrm{Ph}, \mathrm{NR}^{2} \mathrm{R}^{3}=$ Piperidinyl $(68 \%$ yield)

13f; $R=H, R^{1}=E t, N R^{2} R^{3}=$ Piperidinyl $73 \%$ yield)

13g; $R=H, R^{1}=P h, N R^{2} R^{3}=$ Morpholinyl (71\% yield)

13h; $R=C l, R^{1}=P h, N^{2} R^{3}=$ Morpholinyl $(75 \%$ yield $)$

13i; $R=H, R^{1}=E t, N R^{2} R^{3}=$ Morpholinyl $(77 \%$ yield $)$

Scheme 1 Synthetic route towards 4-hydroxyquinazoline-4-carboxamides 13a-i. 

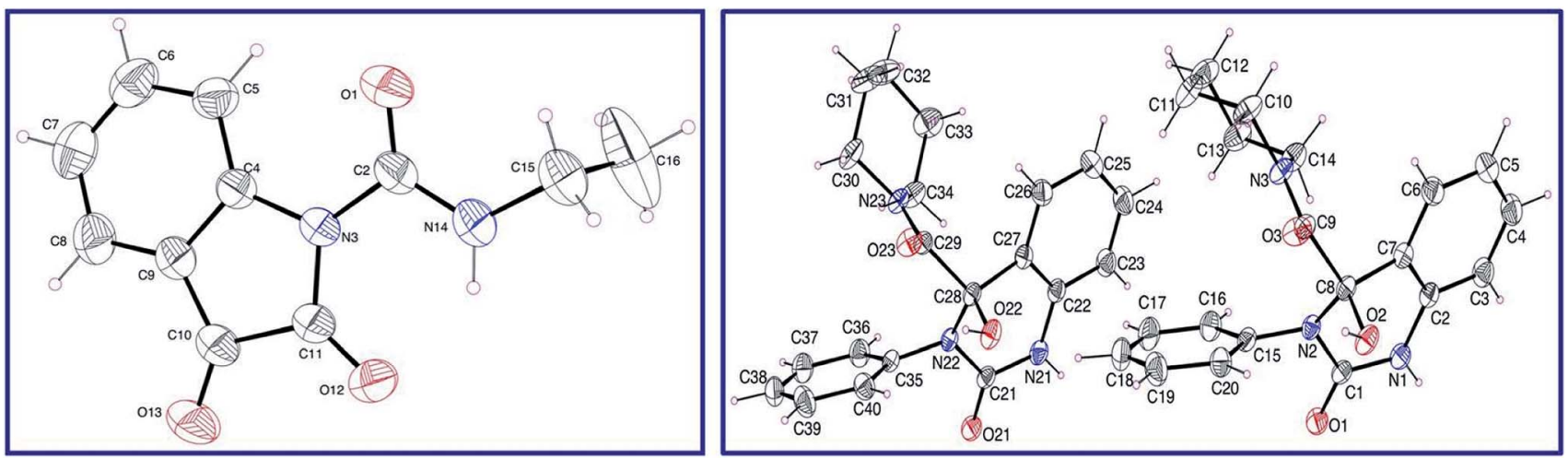

Fig. 2 ORTEP views of compounds $10 \mathrm{c}$ (left) and 13d (right) with the atom-numbering scheme. $\mathrm{H}$ atoms are shown as small spheres of arbitrary radii.

spectrum of 13a (an example of the synthesized analogues) reveals the carbonyl groups as a broad signal at $\nu=$ $1670 \mathrm{~cm}^{-1}$. ${ }^{1} \mathrm{H}-\mathrm{NMR}$ spectrum of 13a reveals the triplet and quartet signals of the ethyl groups at $\delta_{\mathrm{H}}=1.03$ and $2.74 \mathrm{ppm}$, respectively, which are also well observed at $\delta_{\mathrm{C}}=$ $10.8,18.5 ; 41.1,56.0$, respectively in the ${ }^{13} \mathrm{C}$-NMR spectrum. The quinazolinyl C-2 and amidic carbonyls are shown at $\delta_{\mathrm{C}}$ $=151.9,172.4 \mathrm{ppm}$, respectively. Meanwhile, the characteristic quinazolinyl C-4 is exhibited at $\delta_{\mathrm{C}}=87.1 \mathrm{ppm}$ (spectral charts are exhibited in ESI Fig. S1-S30, $\dagger$ overlapping of few carbon signals in the ${ }^{13} \mathrm{C}-\mathrm{NMR}$ spectral data of compounds $\mathbf{1 3 g}, \mathbf{h}$ is observed). Single crystal X-ray studies of 13d support the chemical/stereochemical structure assigned (Fig. 2). The reaction is assumed to take place through amine $\mathbf{1 1}$ nucleophilic attack at the indolyl C-2 giving rise to five-membered ring opening followed by recyclization with ring expansion to the six-membered quinazoline heterocycle (Scheme 1).

\section{X-ray studies}

ORTEP views of compounds 10c and 13d are revealed in Fig. 2. Compound 10c is crystallized in the monoclinic space group $C 2 /$ $c$ while, compound 13d is crystallized in the monoclinic space group $P 2_{1} / n$ (ESI Table S1 $\dagger$ ). The main constituent of $10 \mathrm{c}$ is the indolyl heterocycle and the main constituents of 13d are quinazolinyl, piperidinyl and phenyl rings. Compound 10c exhibits one molecule per asymmetric unit cell and four molecules per unit cell. However, compound 13d shows two molecules per asymmetric unit cell and eight molecules per unit cell. In 10c, the ethyl group is nearly perpendicular to the plane containing the main constituent. Meanwhile, the piperidinyl ring of compound 13d exhibits a chair conformation and the phenyl as well as quinazolinyl heterocycle are nearly planar. Generally, the geometrical parameters of compounds 10c and 13d (ESI Tables $\mathrm{S} 2$ and $\mathrm{S} 3 \dagger)$ are comparable to structures having similar constituents. ${ }^{37}$ Different sets of intermolecular hydrogenbonding interactions stabilize the crystal structure of the studied compounds and led to the formation of supramolecular assemblies (ESI Table S4, Fig. S31 and S32†).

\section{Vasodilation studies}

The standard pre-contracted (norepinephrine hydrochloride) rat aorta technique was utilized for determination the vasodilation properties of the synthesized compounds 10c, 13a-i and compared with Doxazosin $\left(\alpha_{1}\right.$-AR antagonist). ${ }^{38}$ From the exhibited data (Table 1, ESI Fig. S33 and S34†) it can be concluded that, many of the synthesized quinazolines are vasorelaxant active agents with efficacy comparable to that of Doxazosin. Compound 13h seems superior among all the tested analogues with 2.2 folds potency relative to the standard reference $\left(\mathrm{IC}_{50}=158,348 \mu \mathrm{M}\right.$ for $\mathbf{1 3 h}$ and Doxazosin, respectively). Compounds 13 e also shows remarkable vasorelaxant properties (about 139\% potency of Doxazosin with $\left.\mathrm{IC}_{50}=250 \mu \mathrm{M}\right)$. Promising smooth muscle relaxation is also shown by compounds 13a,d,f,g $\left(\mathrm{IC}_{50}=298-332 \mu \mathrm{M}\right)$. $N$-Ethyl-2,3dioxoindoline-1-carboxamide (10c) is also a promising vasorelaxant active agent (1.47 folds efficacy relative to the standard drug).

Based on the observed vasodilation properties few SAR (structure-activity relationship) rules can be stated. The chlorine substituent attached at the quinazolinyl C-6 is an important factor for enhancing the biological properties observed as exhibited in pairs $\mathbf{1 3 b} / \mathbf{1 3 c}, \mathbf{1 3 d} / \mathbf{1 3 e}$ and $\mathbf{1 3 g} / \mathbf{1 3 h}$. The alicyclicamino ring of the amidic function is also a controlling parameter for biological activity. The importance of the amino ring for

Table 1 Vasorelaxant properties of 10c, 13a-i and Doxazosin

\begin{tabular}{llllll}
\hline Entry & Compd. & $\mathrm{R}$ & $\mathrm{R}^{1}$ & $\mathrm{NR}^{2} / \mathrm{R}^{3}$ & $\mathrm{IC}_{50}, \mu \mathrm{M}$ \\
\hline 1 & 10c & $\mathrm{H}$ & $\mathrm{Et}$ & - & 236 \\
2 & 13a & $\mathrm{H}$ & $\mathrm{Ph}$ & $\mathrm{NEt}_{2}$ & 302 \\
3 & $\mathbf{1 3 b}$ & $\mathrm{H}$ & $\mathrm{Ph}$ & Pyrrolidinyl $^{2}$ & 415 \\
4 & 13c & $\mathrm{Cl}$ & $\mathrm{Ph}$ & Pyrrolidinyl & 392 \\
5 & 13d & $\mathrm{H}$ & $\mathrm{Ph}$ & Piperidinyl & 332 \\
6 & 13e & $\mathrm{Cl}$ & $\mathrm{Ph}$ & Piperidinyl & 250 \\
7 & 13f & $\mathrm{H}$ & $\mathrm{Et}$ & Piperidinyl & 305 \\
8 & $\mathbf{1 3 g}$ & $\mathrm{H}$ & $\mathrm{Ph}$ & Morpholinyl & 298 \\
9 & $\mathbf{1 3 h}$ & $\mathrm{Cl}$ & $\mathrm{Ph}$ & Morpholinyl & 158 \\
10 & $\mathbf{1 3 i}$ & $\mathrm{H}$ & $\mathrm{Et}$ & Morpholinyl & 416 \\
11 & Doxazosin & - & - & - & 348
\end{tabular}


vasodilation properties enhancement can be arranged in the following order morpholinyl $>$ piperidinyl $>$ pyrrolidinyl (compound 13f is an exception).

\section{Molecular modeling studies}

2D-QSAR study. QSAR (quantitative structure-activity relationship) study is a widely used computational technique for explaining the biological observations and better understanding the parameters optimizing properties. $^{39}$ Two descriptor QSAR model was obtained by CODESSA-Pro for the vasorelaxant quinazoline-4-carboxamides 13a-i $\left(R^{2}=0.970\right.$, ESI Table S5 $\dagger$ ). Explanation/calculation of the QSAR descriptors was mentioned in the ESI. $\dagger$ Goodness of the QSAR model is supported by the leave one-out and leave many-out (up to $20 \%$ of the compounds used in the study) coefficient values relative to the original QSAR coefficient value $\left(R^{2}=0.970, R^{2} \mathrm{cvOO}=0.905\right.$, $R^{2}$ cvMO $\left.=0.937\right)$. The predicted $\mathrm{IC}_{50}$ values relative to the experimental are also supporting evidence for the robust of computational model (Fig. 3).

3D-Pharmacophore study. Three chemical features (two hydrophobics " $\mathrm{H}-1, \mathrm{H}-2$ " and one hydrogen bonding donor "HBD", ESI Fig. S35 and S36†) are shown by the 3Dpharmacophoric model (Discovery Studio 2.5 software) for the vasorelaxant active quinazoline-4-carboxamides $13 a-i .{ }^{40}$ All the synthesized compounds reveal alignment of the hydroxyl group with the HBD and the amino residue/ring of the amidic linkage with one of the hydrophobic in variable fitness affording diverse estimated biological properties (ESI Table S8 $\dagger$ ). These observations support the elements of design for the present study and also the assigned SAR mentioned. The high correlations between the estimated and

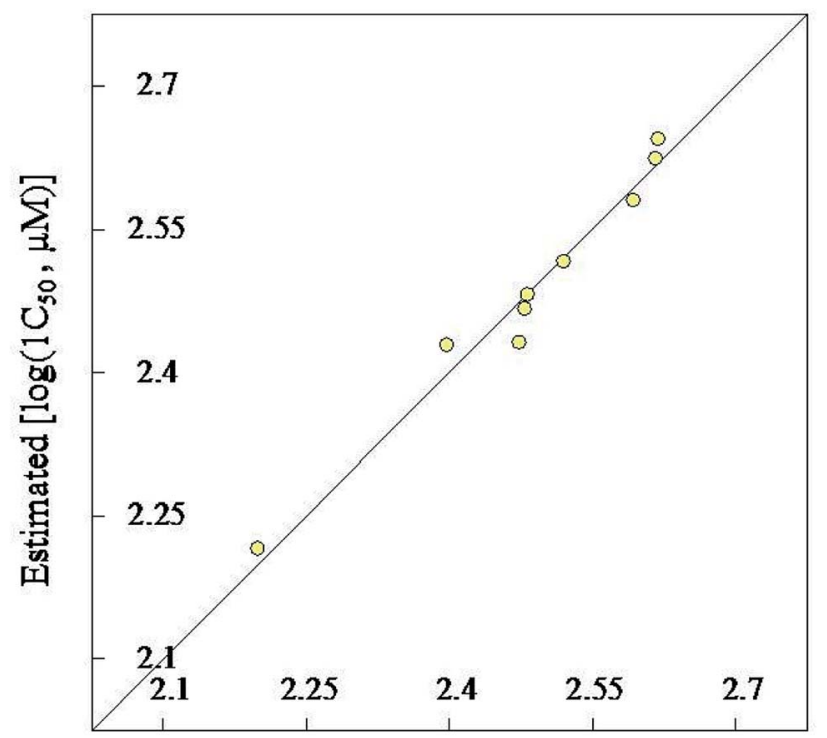

\section{Observed $\left[\log \left(1 \mathrm{C}_{50}, \mu \mathrm{M}\right)\right]$}

Fig. 3 BMLR-QSAR model plot of correlation representing the observed versus predicted $\left[\log \left(I_{50}, \mu M\right)\right]$ values for the synthesized vasorelaxant quinazoline (13a-i). experimental biological properties support the goodness of the 3D-pharmacophoric model.

\section{Conclusions}

4-Hydroxyquinazoline-4-carboxamides $13 \mathbf{a}-\mathbf{i}$ are synthesized in a facile synthetic approach (yield 68-85\%) via reaction of 2,3dioxoindoline-1-carboxamides 10a-c with secondary amines 11a-d in dry THF. Single crystal X-ray studies of 10c and 13d support the chemical structures. Some of the synthesized quinazolines reveal vasorelaxant properties with efficacy comparable to that of Doxazosin especially, compound $\mathbf{1 3 h}$ that exhibits 2.2 folds potency relative to the standard reference. 2DQSAR model describes the biological observations $(N=9, n=2$, $R^{2}=0.970$ ). Also, the 3D-pharmacophoric model supports the elements of design for the present study.

\section{Experimental}

\section{Chemistry}

Melting points were determined on a capillary point apparatus (Stuart SMP3) equipped with a digital thermometer. IR spectra (KBr) were recorded on a Shimadzu FT-IR 8400S spectrophotometer. Reactions were monitored using thin layer chromatography (TLC) on $0.2 \mathrm{~mm}$ silica gel F254 plates (Merck) utilizing various solvents for elution. The chemical structures of the synthesized compounds were characterized by nuclear magnetic resonance spectra $\left({ }^{1} \mathrm{H}-\mathrm{NMR},{ }^{13} \mathrm{C}-\mathrm{NMR}\right)$ and determined on a Bruker NMR spectrometer $(500 \mathrm{MHz}, 125 \mathrm{MHz}) \cdot{ }^{13} \mathrm{C}$ NMR spectra are fully decoupled. Chemical shifts were reported in parts per million (ppm) using the deuterated solvent peak or tetramethylsilane as an internal standard.

\section{Reaction of $8 a, b$ with isocyanates $9 a, b$}

A solution of the corresponding isocyanate $\mathbf{9 a}, \mathbf{b}$ ( $5 \mathrm{mmol})$ in dry THF ( $5 \mathrm{ml}$ ) was added dropwise to a mixture of the appropriate 8a,b (5 mmol) in dry THF (20 ml) containing triethylamine (5.5 $\mathrm{mmol}$ ), at $0{ }^{\circ} \mathrm{C}$. The reaction was magnetically stirred at the same temperature for $10 \mathrm{~h}$ then, stored in the fridge overnight. The separated solid was collected and washed with benzene affording 10a,b which was used without any more purifications. In case of the reaction of $\mathbf{8 a}$ and $\mathbf{9 b}$, the separated solid was crystallized from benzene affording 10c as orange microcrystals.

$\boldsymbol{N}$-Ethyl-2,3-dioxoindoline-1-carboxamide (10c). It was obtained from the reaction of $\mathbf{8 a}$ and $\mathbf{9 b}$ (reaction time $10 \mathrm{~h}$ ) as orange microcrystals from benzene with $\mathrm{mp} 159-161{ }^{\circ} \mathrm{C}$ and yield 73\% (0.80 g). IR: $\nu_{\max } / \mathrm{cm}^{-1} 3337,1756,1740,1701,1612$. ${ }^{1} \mathrm{H}-\mathrm{NMR}$ (DMSO- $\left.d_{6}\right) \delta(\mathrm{ppm}): 1.15\left(\mathrm{t}, J=7.2 \mathrm{~Hz}, 3 \mathrm{H}, \mathrm{CH}_{3} \mathrm{CH}_{2}\right.$ ), 3.30-3.35 (m, 2H, $\left.\mathrm{CH}_{3} \mathrm{CH}_{2}\right), 7.28$ (t, $J=7.5 \mathrm{~Hz}, 1 \mathrm{H}$, arom. $\mathrm{H}$ ), 7.67 (d, $J=7.5 \mathrm{~Hz}, 1 \mathrm{H}$, arom. H), $7.71(\mathrm{t}, J=7.9 \mathrm{~Hz}, 1 \mathrm{H}$, arom. $\mathrm{H}), 8.17(\mathrm{~d}, J=8.2 \mathrm{~Hz}, 1 \mathrm{H}$, arom. $\mathrm{H}), 8.22(\mathrm{t}, J=5.3 \mathrm{~Hz}, 1 \mathrm{H}, \mathrm{NH})$. ${ }^{13} \mathrm{C}-\mathrm{NMR}$ (DMSO- $\left.d_{6}\right) \delta(\mathrm{ppm}): 14.7\left(\mathrm{CH}_{2} \mathrm{CH}_{3}\right), 34.4\left(\mathrm{NCH}_{2} \mathrm{CH}_{3}\right)$, 116.5, 118.9, 124.3, 124.6, 137.5, 148.2 (arom. C), 150.4 (urea CO), 158.9 (indolyl C-2), 180.4 (indolyl C-3). Anal. calcd for 
$\mathrm{C}_{11} \mathrm{H}_{10} \mathrm{~N}_{2} \mathrm{O}_{3}$ (218.21): C, 60.55; H, 4.62; N, 12.84. Found: $\mathrm{C}$, 60.76; H, 4.77; N, 12.93 .

\section{Reaction of 10a-c with secondary amines 11a-d}

The appropriate secondary amine 11a-d (5.5 mmol) was added dropwise to the magnetically stirred solution of $10 a-c(5 \mathrm{mmol})$ in dry THF $(20 \mathrm{ml})$ at room temperature $\left(20-25{ }^{\circ} \mathrm{C}\right)$ for the appropriate time. The separated solid was collected and crystallized from a suitable solvent affording the corresponding $\mathbf{1 3 a}, \mathbf{c}, \mathbf{d}, \mathbf{g}-\mathbf{i}$. In case of $\mathbf{1 3 b}, \mathbf{1 3 e}$, and $\mathbf{1 3 f}$ the reaction mixture was evaporated till dryness under reduced pressure. The remaining material was triturated with methanol $(5 \mathrm{ml})$. The separated solid was collected and crystallized from a suitable solvent.

$N, N$-Diethyl-4-hydroxy-2-oxo-3-phenyl-1,2,3,4-tetrahydroquinazoline4-carboxamide (13a). It was obtained from the reaction of 10a and 11a (reaction time $8 \mathrm{~h}$ ) as colorless microcrystals from methanol with $\mathrm{mp}$ $145-147{ }^{\circ} \mathrm{C}$ and yield $71 \%$ (1.20 g). IR: $\nu_{\max } / \mathrm{cm}^{-1} 3202,3059,1670$, 1609, 1497, 1412. ${ }^{1} \mathrm{H}-\mathrm{NMR}$ (DMSO- $\left.d_{6}\right) \delta(\mathrm{ppm}): 1.03(\mathrm{t}, J=6.9 \mathrm{~Hz}, 6 \mathrm{H}, 2$ $\mathrm{CH}_{2} \mathrm{CH}_{3}$ ), 2.74 (q, $=6.4 \mathrm{~Hz}, 4 \mathrm{H}, 2 \mathrm{CH}_{2} \mathrm{CH}_{3}$ ), 6.41 (br s, $\left.1 \mathrm{H}, \mathrm{NH}\right), 6.80$ 6.85 (m, 2H, arom. H), 7.05 (d, $J=7.4 \mathrm{~Hz}, 1 \mathrm{H}$, arom. H), 7.14 (t, $J=$ $7.3 \mathrm{~Hz}, 1 \mathrm{H}$, arom. H), 7.22-7.27 (m, 5H, arom. H), 9.62 (br s, $1 \mathrm{H}, \mathrm{OH}$ ). ${ }^{13} \mathrm{C}-\mathrm{NMR}$ (DMSO- $\left.d_{6}\right) \delta$ (ppm): $10.8\left(\mathrm{CH}_{2} \mathrm{CH}_{3}\right), 18.5\left(\mathrm{CH}_{2} \mathrm{CH}_{3}\right), 41.1$ $\left(\mathrm{NCH}_{2} \mathrm{CH}_{3}\right), 56.0\left(\mathrm{NCH}_{2} \mathrm{CH}_{3}\right), 87.1$ (quinazolinyl C-4), 113.0, 120.5, 124.0, 125.7, 126.5, 127.6, 128.1, 130.7, 136.0, 139.3 (arom. C), 151.9 (quinazolinyl C-2), 172.4 (carboxamide CO). Anal. calcd for $\mathrm{C}_{19} \mathrm{H}_{21} \mathrm{~N}_{3} \mathrm{O}_{3}$ (339.40): C, 67.24; H, 6.24; N, 12.38. Found: C, 67.38; H, 6.30; N, 12.42.

4-Hydroxy-3-phenyl-4-(pyrrolidine-1-carbonyl)-3,4-dihydroquinazolin-2(1H)-one $(\mathbf{1 3 b})$. It was obtained from the reaction of 10a and $\mathbf{1 1 b}$ (reaction time $10 \mathrm{~h}$ ) as colorless microcrystals from methanol with $\mathrm{mp} 184-186^{\circ} \mathrm{C}$ and yield $74 \%$ (1.25 g). IR: $\nu_{\max } /$ $\mathrm{cm}^{-1}$ 3503, 3402, 1678, 1636, 1605, 1504. ${ }^{1} \mathrm{H}-\mathrm{NMR}$ (DMSO- $d_{6}$ ) $\delta$ (ppm): 1.59-1.76 (m, 3H, pyrrolidinyl $\mathrm{H}), 2.53-2.56(\mathrm{~m}, 1 \mathrm{H}$, pyrrolidinyl H), 3.01 (quintet, $J=7.0 \mathrm{~Hz}, 1 \mathrm{H}$, pyrrolidinyl $\mathrm{H}$ ), $3.18(\mathrm{~d}, J=$ $4.8 \mathrm{~Hz}, 1 \mathrm{H}$, pyrrolidinyl H), 3.35-3.38 (m, 1H, pyrrolidinyl H), 3.48$3.53(\mathrm{~m}, 1 \mathrm{H}$, pyrrolidinyl $\mathrm{H}), 6.70(\mathrm{~s}, 1 \mathrm{H}, \mathrm{NH}), 6.93-7.00(\mathrm{~m}, 3 \mathrm{H}$, arom. H), 7.24 (d, J= 7.4 Hz, 2H, arom. H), 7.30-7.38 (m, 4H, arom. $\mathrm{H}), 10.22$ (s, 1H, OH). ${ }^{13} \mathrm{C}-\mathrm{NMR}$ (DMSO- $\left.d_{6}\right) \delta$ (ppm): 22.7, 25.8 (pyrrolidinyl C-3/4), 46.5, 47.5 (pyrrolidinyl C-2/5), 85.9 (quinazolinyl C-4), 113.8, 119.8, 121.7, 125.5, 127.3, 128.2, 129.4, 129.5, 135.8, 137.6 (arom. C), 150.9 (quinazolinyl C-2), 166.2 (carboxamide CO). Anal. calcd for $\mathrm{C}_{19} \mathrm{H}_{19} \mathrm{~N}_{3} \mathrm{O}_{3}$ (337.38): C, 67.64; H, 5.68; N, 12.46. Found: C, 67.81; H, 5.59; N, 12.57 .

6-Chloro-4-hydroxy-3-phenyl-4-(pyrrolidine-1-carbonyl)-3,4dihydroquinazolin-2(1H)-one (13c). It was obtained from the reaction of $\mathbf{1 0 b}$ and $\mathbf{1 1 b}$ (reaction time $6 \mathrm{~h}$ ) as colorless microcrystals from $n$-butanol with $\mathrm{mp} 208-210{ }^{\circ} \mathrm{C}$ and yield $85 \%$ (1.58 g). IR: $\nu_{\max } / \mathrm{cm}^{-1} 3564,3345,3210,1682,1643,1605,1489 .{ }^{1} \mathrm{H}-$ NMR (DMSO- $\left.d_{6}\right) \delta(\mathrm{ppm}):$ 1.59-1.74 $(\mathrm{m}, 4 \mathrm{H}$, pyrrolidinyl $\mathrm{H})$, 2.57-2.62 (m, 1H, pyrrolidinyl H), 3.01 (quintet, $J=6.7 \mathrm{~Hz}, 1 \mathrm{H}$, pyrrolidinyl $\mathrm{H}), 3.33-3.52(\mathrm{~m}, 2 \mathrm{H}$, pyrrolidinyl $\mathrm{H}), 6.91(\mathrm{~s}, 1 \mathrm{H}$, $\mathrm{NH}), 6.92(\mathrm{~d}, J=2.1 \mathrm{~Hz}, 1 \mathrm{H}$, arom. H), $6.98(\mathrm{~d}, J=8.7 \mathrm{~Hz}, 1 \mathrm{H}$, arom. H), 7.22 (d, $J=7.4 \mathrm{~Hz}, 2 \mathrm{H}$, arom. $\mathrm{H}), 7.31-7.39(\mathrm{~m}, 4 \mathrm{H}$, arom. H), 10.25 (s, $1 \mathrm{H}, \mathrm{OH}) .{ }^{13} \mathrm{C}-\mathrm{NMR}$ (DMSO- $\left.d_{6}\right) \delta$ (ppm): 22.6, 25.9 (pyrrolidinyl C-3/4), 46.6, 47.6 (pyrrolidinyl C-2/5), 86.0 (quinazolinyl C-4), 115.8, 122.0, 124.9, 125.1, 127.4, 128.2, 129.5, 129.6, 134.8, 137.4 (arom. C), 150.6 (quinazolinyl C-2), 165.8 (carboxamide CO). Anal. calcd for $\mathrm{C}_{19} \mathrm{H}_{18} \mathrm{ClN}_{3} \mathrm{O}_{3}$ (371.82): C, 61.38; H, 4.88; N, 11.30. Found: C, 61.57; H, 5.00; N, 11.38.

4-Hydroxy-3-phenyl-4-(piperidine-1-carbonyl)-3,4-dihydroquinazolin-2(1H)-one (13d). It was obtained from the reaction of $10 a$ and $11 \mathrm{c}$ (reaction time $6 \mathrm{~h}$ ) as colorless microcrystals from ethanol with $\mathrm{mp} 176-178{ }^{\circ} \mathrm{C}$ and yield $77 \%$ (1.35 g). IR: $\nu_{\max } / \mathrm{cm}^{-1} 3387,1682,1636,1605,1501 .{ }^{1} \mathrm{H}-$ NMR (DMSO- $\left.d_{6}\right) \delta(\mathrm{ppm}): 0.62$ (br s, $1 \mathrm{H}$, piperidinyl H), 1.12$1.46(\mathrm{~m}, 5 \mathrm{H}$, piperidinyl $\mathrm{H}), 2.64(\mathrm{t}, J=10.9 \mathrm{~Hz}, 1 \mathrm{H}$, piperidinyl $\mathrm{H}), 3.12(\mathrm{t}, J=11.8 \mathrm{~Hz}, 1 \mathrm{H}$, piperidinyl $\mathrm{H}), 3.49(\mathrm{~d}, J=$ $12.8 \mathrm{~Hz}, 1 \mathrm{H}$, piperidinyl $\mathrm{H}$ ), $3.94(\mathrm{~d}, J=12.3 \mathrm{~Hz}, 1 \mathrm{H}$, piperidinyl H), 6.75 (s, 1H, NH), 6.93-7.00 (m, 3H, arom. H), 7.30$7.34\left(\mathrm{~m}, 6 \mathrm{H}\right.$, arom. H) $10.22(\mathrm{~s}, 1 \mathrm{H}, \mathrm{OH}) .{ }^{13} \mathrm{C}-\mathrm{NMR}$ (DMSO$\left.d_{6}\right) \delta(\mathrm{ppm}): 23.3,24.0,25.1$ (piperidinyl C-3/4/5), 44.3, 46.9 (piperidinyl C-2/6), 85.4 (quinazolinyl C-4), 114.2, 120.6, 121.7, 125.6, 127.5, 128.3, 129.5, 129.7, 135.0, 137.8 (arom. C), 150.7 (quinazolinyl C-2), 166.2 (carboxamide CO). Anal. calcd for $\mathrm{C}_{20} \mathrm{H}_{21} \mathrm{~N}_{3} \mathrm{O}_{3}$ (351.41): C, 68.36; H, 6.02; N, 11.96. Found: C, 68.14; H, 5.84; N, 12.16.

6-Chloro-4-hydroxy-3-phenyl-4-(piperidine-1-carbonyl)-3,4dihydroquinazolin-2(1H)-one (13e). It was obtained from the reaction of $10 \mathrm{~b}$ and $11 \mathrm{c}$ (reaction time $12 \mathrm{~h}$ ) as colorless microcrystals from $n$-butanol of mp $180-182{ }^{\circ} \mathrm{C}$ and yield $68 \%$ (1.30 g). IR: $\nu_{\max } / \mathrm{cm}^{-1} 3314,3210,1682,1643,1605,1493 .{ }^{1} \mathrm{H}-$ NMR (DMSO- $\left.d_{6}\right) \delta(\mathrm{ppm}): 0.65-0.77(\mathrm{~m}, 1 \mathrm{H}$, piperidinyl $\mathrm{H})$, 1.22-1.53 (m, 5H, piperidinyl $\mathrm{H}), 2.69(\mathrm{t}, J=11.0 \mathrm{~Hz}, 1 \mathrm{H}$, piperidinyl $\mathrm{H}), 3.16(\mathrm{t}, J=11.4 \mathrm{~Hz}, 1 \mathrm{H}$, piperidinyl $\mathrm{H}), 3.48(\mathrm{~d}, J$ $=13.4 \mathrm{~Hz}, 1 \mathrm{H}$, piperidinyl $\mathrm{H}), 3.93(\mathrm{~d}, J=12.5 \mathrm{~Hz}, 1 \mathrm{H}$, piperidinyl H), 6.90 (br s, 2H, NH + arom. H), 7.01 (d, $J=8.6 \mathrm{~Hz}, 1 \mathrm{H}$, arom. H), 7.30-7.42 (m, 6H, arom. H), 10.45 (s, $1 \mathrm{H}, \mathrm{OH}) .{ }^{13} \mathrm{C}-$ NMR (DMSO- $d_{6}$ ) $\delta$ (ppm): 23.2, 24.1, 25.1 (piperidinyl C-3/4/5), 44.1, 46.7 (piperidinyl C-2/6), 85.1 (quinazolinyl C-4), 116.0, 122.3, 124.86, 124.94, 127.4, 128.2, 129.3, 129.6, 134.0, 137.5 (arom. C), 150.4 (quinazolinyl C-2), 165.5 (carboxamide CO). Anal. calcd for $\mathrm{C}_{20} \mathrm{H}_{20} \mathrm{ClN}_{3} \mathrm{O}_{3}$ (385.85): C, 62.26; H, 5.22; N, 10.89. Found: C, 62.44; H, 5.03; N, 10.73.

3-Ethyl-4-hydroxy-4-(piperidine-1-carbonyl)-3,4-dihydroquinazolin-2(1H)-one (13f). It was obtained from the reaction of 10c and 11c (reaction time $24 \mathrm{~h}$ ) as colorless microcrystals from benzene with $\mathrm{mp} 165-167{ }^{\circ} \mathrm{C}$ and yield $73 \%(1.10 \mathrm{~g})$. IR: $\nu_{\max } / \mathrm{cm}^{-1} 3318,3198,3121,1667,1636$, 1609, 1504. ${ }^{1} \mathrm{H}-\mathrm{NMR}$ (DMSO- $\left.d_{6}\right) \delta(\mathrm{ppm}): 0.61$ (br s, $1 \mathrm{H}$, piperidinyl $\mathrm{H}), 1.10\left(\mathrm{t}, J=7.0 \mathrm{~Hz}, 4 \mathrm{H}, \mathrm{CH}_{3} \mathrm{CH}_{2}+\right.$ piperidinyl $\mathrm{H})$, 1.37-1.53 (m, 4H, piperidinyl $\mathrm{H}), 3.00-3.33(\mathrm{~m}, 5 \mathrm{H}$, $\mathrm{CH}_{3} \mathrm{CH}_{2}+3$ piperidinyl $\mathrm{H}$ ), 3.96 (br s, $1 \mathrm{H}$, piperidinyl $\mathrm{H}$ ), $6.75(\mathrm{~s}, 1 \mathrm{H}, \mathrm{NH}), 6.86(\mathrm{~d}, J=8.1 \mathrm{~Hz}, 1 \mathrm{H}$, arom. H), 6.90-6.96 $(\mathrm{m}, 2 \mathrm{H}$, arom. H), $7.25(\mathrm{t}, J=7.6 \mathrm{~Hz}, 1 \mathrm{H}$, arom. H), $9.96(\mathrm{~s}$, $1 \mathrm{H}, \mathrm{OH}) .{ }^{13} \mathrm{C}-\mathrm{NMR}\left(\mathrm{DMSO}-d_{6}\right) \delta(\mathrm{ppm}): 14.3\left(\mathrm{CH}_{3} \mathrm{CH}_{2}\right), 23.2$, 24.1, 25.1 (piperidinyl C-3/4/5), $37.8\left(\mathrm{CH}_{3} \mathrm{CH}_{2}\right), 44.5,46.5$ (piperidinyl C-2/6), 84.6 (quinazolinyl C-4), 113.6, 119.6, 121.3, 125.7, 129.5, 135.0 (arom. C), 150.3 (quinazolinyl C2), 167.5 (carboxamide CO). Anal. calcd for $\mathrm{C}_{16} \mathrm{H}_{21} \mathrm{~N}_{3} \mathrm{O}_{3}$ (303.36): C, 63.35; H, 6.98; N, 13.85. Found: C, 63.22; H, $6.87 ; \mathrm{N}, 13.79$.

4-Hydroxy-4-(morpholine-4-carbonyl)-3-phenyl-3,4-dihydroquinazolin-2(1H)-one $(\mathbf{1 3 g})$. It was obtained from the reaction of 10a and 11d (reaction time $20 \mathrm{~h}$ ) as colorless microcrystals from 
ethanol with mp 173-175 ${ }^{\circ} \mathrm{C}$ and yield $71 \%$ (1.25 g). IR: $\nu_{\max } / \mathrm{cm}^{-1}$ 3618, 3526, 3345, 3198, 1682, 1639, 1609. ${ }^{1} \mathrm{H}-\mathrm{NMR}$ (DMSO- $d_{6}$ ) $\delta(\mathrm{ppm}): 2.83(\mathrm{t}, J=8.9 \mathrm{~Hz}, 1 \mathrm{H}$, morpholinyl $\mathrm{H}), 2.96(\mathrm{t}, J=9.5 \mathrm{~Hz}$, 1H, morpholinyl $\mathrm{H}$ ), 3.20-3.45 (m, 5H, morpholinyl $\mathrm{H}$ ), 3.65-3.72 (m, 1H, morpholinyl H), 6.79 (s, 1H, NH), 6.96-7.03 (m, 3H, arom. H), 7.29-7.35 (m, 6H, arom. H), 10.28 (s, 1H, OH). ${ }^{13}$ C-NMR (DMSO$\left.d_{6}\right) \delta$ (ppm): 43.4, 46.7 (morpholinyl $\mathrm{NCH}_{2}$ ), 64.6, 65.7 (morpholinyl $\mathrm{OCH}_{2}$ ), 85.8 (quinazolinyl C-4), 114.2, 118.7, 120.7, 121.6, 125.3, 127.2 128.1, 128.7, 129.2, 129.6, 135.0, 137.9 (arom. C), 150.8 (quinazolinyl C-2), 166.5 (carboxamide CO). Anal. calcd for $\mathrm{C}_{19} \mathrm{H}_{19} \mathrm{~N}_{3} \mathrm{O}_{4}$ (353.38): C, 64.58; H, 5.42; N, 11.89. Found: C, 64.64; H, 5.56; N, 11.76.

6-Chloro-4-hydroxy-4-(morpholine-4-carbonyl)-3-phenyl-3,4dihydroquinazolin-2(1H)-one $(\mathbf{1 3 h})$. It was obtained from the reaction of 10b and 11d (reaction time $6 \mathrm{~h}$ ) as colorless microcrystals from $n$-butanol with mp $189-191{ }^{\circ} \mathrm{C}$ and yield $75 \%(1.45$ g). IR: $\nu_{\max } / \mathrm{cm}^{-1} 3352,3213,1686,1643,1609 .{ }^{1} \mathrm{H}-\mathrm{NMR}$ (DMSO$\left.d_{6}\right) \delta(\mathrm{ppm}): 2.82-2.86(\mathrm{~m}, 1 \mathrm{H}$, morpholinyl $\mathrm{H}), 2.99(\mathrm{t}, J=$ $9.1 \mathrm{~Hz}, 1 \mathrm{H}$, morpholinyl $\mathrm{H}), 3.23-3.43(\mathrm{~m}, 5 \mathrm{H}$, morpholinyl $\mathrm{H})$, 3.64 (br d, $J=10.1 \mathrm{~Hz}, 1 \mathrm{H}$, morpholinyl H), 6.95-7.02 (m, 3H, $\mathrm{NH}+2$ arom. $\mathrm{H}), 7.31-7.41(\mathrm{~m}, 6 \mathrm{H}$, arom. $\mathrm{H}), 10.42(\mathrm{~s}, 1 \mathrm{H}, \mathrm{OH})$. ${ }^{13}$ C-NMR (DMSO- $d_{6}$ ) $\delta$ (ppm): 43.4, 46.7 (morpholinyl $\mathrm{NCH}_{2}$ ), 64.8, 65.8 (morpholinyl $\mathrm{OCH}_{2}$ ), 85.8 (quinazolinyl C-4), 116.1, 124.6, 125.0, 127.3, 128.2, 129.2, 129.6, 134.1, 137.7 (arom. C), 150.5 (quinazolinyl C-2), 166.0 (carboxamide $\mathrm{CO}$ ). Anal. calcd for $\mathrm{C}_{19} \mathrm{H}_{18} \mathrm{ClN}_{3} \mathrm{O}_{4}$ (387.82): C, 58.84; H, 4.68; N, 10.84. Found: C, 58.96; H, 4.78; N, 11.04.

3-Ethyl-4-hydroxy-4-(morpholine-4-carbonyl)-3,4-dihydroquinazolin-2(1H)-one (13i). It was obtained from the reaction of $10 \mathrm{c}$ and $\mathbf{1 1 d}$ (reaction time $10 \mathrm{~h}$ ) as colorless microcrystals from benzene with $\mathrm{mp} 161-163{ }^{\circ} \mathrm{C}$ and yield $77 \%$ (1.17 g). IR: $\nu_{\max } / \mathrm{cm}^{-1} 3310,3267,3210,1748,1655$, 1609. ${ }^{1} \mathrm{H}-\mathrm{NMR}\left(\mathrm{CDCl}_{3}\right) \delta(\mathrm{ppm}): 1.33(\mathrm{t}, J=7.0 \mathrm{~Hz}, 3 \mathrm{H}$, $\mathrm{CH}_{3} \mathrm{CH}_{2}$ ), 2.94 (br t, $J=9.8 \mathrm{~Hz}, 1 \mathrm{H}$, morpholinyl H), 3.11 (br $\mathrm{d}, J=13.7 \mathrm{~Hz}, 1 \mathrm{H}$, morpholinyl $\mathrm{H}), 3.23-3.27$ (m, $1 \mathrm{H}$, morpholinyl $\mathrm{H}), 3.34-3.55\left(\mathrm{~m}, 4 \mathrm{H}, \mathrm{CH}_{3} \mathrm{CH}_{2}+2\right.$ morpholinyl $\left.\mathrm{H}\right)$, $3.61(\mathrm{t}, J=9.8 \mathrm{~Hz}, 1 \mathrm{H}$, morpholinyl $\mathrm{H}), 3.78(\mathrm{br} \mathrm{d}, J=11.9 \mathrm{~Hz}$, 1H, morpholinyl $\mathrm{H}$ ), 4.01 (br d, $J=13.3 \mathrm{~Hz}, 1 \mathrm{H}$, morpholinyl $\mathrm{H}), 6.65(\mathrm{~s}, 1 \mathrm{H}, \mathrm{NH}), 6.87(\mathrm{~d}, J=8.1 \mathrm{~Hz}, 1 \mathrm{H}$, arom. H), $7.03(\mathrm{t}, J$ $=7.4 \mathrm{~Hz}, 1 \mathrm{H}$, arom. $\mathrm{H}), 7.06(\mathrm{t}, J=6.5 \mathrm{~Hz}, 1 \mathrm{H}$, arom. $\mathrm{H}), 7.31$ (d, $J=8.3 \mathrm{~Hz}, 1 \mathrm{H}$, arom. H), 9.57 (s, 1H, OH). ${ }^{13} \mathrm{C}-\mathrm{NMR}$ $\left(\mathrm{CDCl}_{3}\right) \delta$ (ppm): $14.7\left(\mathrm{CH}_{3} \mathrm{CH}_{2}\right), 38.8\left(\mathrm{CH}_{3} \mathrm{CH}_{2}\right), 44.6,46.9$ (morpholinyl $\mathrm{NCH}_{2}$ ), 65.3, 66.4 (morpholinyl $\mathrm{OCH}_{2}$ ), 85.0 (quinazolinyl C-4), 114.4, 119.1, 122.6, 125.9, 130.3, 134.5 (arom. C), 152.0 (quinazolinyl C-2), 169.1 (carboxamide CO). Anal. calcd for $\mathrm{C}_{15} \mathrm{H}_{19} \mathrm{~N}_{3} \mathrm{O}_{4}$ (305.33): C, 59.01; H, 6.27; N, 13.76. Found: C, 59.08; H, 6.36; N, 13.89 .

\section{Single crystal X-ray studies}

Experimental procedure for the single X-ray studies of compounds 10c and 13d is mentioned in the ESI. $\dagger$

\section{Vasodilation studies}

Experimental procedure utilized for vasodilation studies is mentioned in the ESI. $\dagger$

\section{Molecular modeling studies}

Experimental procedures for the molecular modeling studies are mentioned in the ESI. $\dagger$

\section{Conflicts of interest}

The authors have declared no conflict of interest.

\section{Acknowledgements}

This work was supported financially by National Research Centre, Egypt, project ID: 11010341.

\section{Notes and references}

1 D. Das and J. Hong, Recent advancements of 4aminoquinazoline derivatives as kinase inhibitors and their applications in medicinal chemistry, Eur. J. Med. Chem., 2019, 170, 55-72.

2 V. Alagarsamy, K. Chitra, G. Saravanan, V. R. Solomon, M. T. Sulthana and B. Narendhar, An overview of quinazolines: pharmacological significance and recent developments, Eur. J. Med. Chem., 2018, 151, 628-685.

3 I. Khan, S. Zaib, S. Batool, N. Abbas, Z. Ashraf, J. Iqbal and A. Saeed, Quinazolines and quinazolinones as ubiquitous structural fragments in medicinal chemistry: an update on the development of synthetic methods and pharmacological diversification, Bioorg. Med. Chem., 2016, 24, 2361-2381.

4 I. Khan, A. Ibrar, W. Ahmed and A. Saeed, Synthetic approaches, functionalization and therapeutic potential of quinazoline and quinazolinone skeletons: the advances continue, Eur. J. Med. Chem., 2015, 90, 124-169.

5 S. Ravez, O. Castillo-Aguilera, P. Depreux and L. Goossens, Quinazoline derivatives as anticancer drugs: a patent review (2011 - present), Expert Opin. Ther. Pat., 2015, 25, 789-804.

6 I. Khan, A. Ibrar, N. Abbas and A. Saeed, Recent advances in the structural library of functionalized quinazoline and quinazolinone scaffolds: synthetic approaches and multifarious applications, Eur. J. Med. Chem., 2014, 76, 193-244.

7 https://www.drugs.com/history/iressa.html.

8 https://www.cancer.gov/about-cancer/treatment/drugs/ gefitinib.

9 https://www.drugs.com/history/tarceva.html.

10 https:/www.cancer.gov/about-cancer/treatment/drugs/ erlotinibhydrochloride.

11 https://www.drugs.com/history/tykerb.html.

12 https:/www.cancer.gov/about-cancer/treatment/drugs/ lapatinibditosylate.

13 Y. Zhang, L. Chen, H. Xu, X. Li, L. Zhao, W. Wang, B. Li and X. Zhang, 6,7-Dimorpholinoalkoxy quinazoline derivatives as potent EGFR inhibitors with enhanced antiproliferative activities against tumor cells, Eur. J. Med. Chem., 2018, 147, 77-89. 
14 https://www.drugs.com/history/gilotrif.html.

15 https://www.cancer.gov/about-cancer/treatment/drugs/ afatinibdimaleate.

16 https://www.drugs.com/history/vizimpro.html.

17 https://www.cancer.gov/about-cancer/treatment/drugs/ dacomitinib.

18 G. Romeo, L. Materia, G. Marucci, M. Modica, V. Pittalà, L. Salerno, M. A. Siracusa, M. Buccioni, P. Angeli and K. P. Minneman, New pyrimido[5,4- $b]$ indoles and [1] benzothieno $[3,2-d]$ pyrimidines: high affinity ligands for the $\alpha_{1}$-adrenoceptor subtypes, Bioorg. Med. Chem. Lett., 2006, 16, 6200-6203.

19 https://www.who.int/cardiovascular_diseases/en/.

20 https://www.who.int/cardiovascular_diseases/world-heartday/en/.

21 K. S. Jain, J. B. Bariwal, M. K. Kathiravan, M. S. Phoujdar, R. S. Sahne, B. S. Chauhan, A. K. Shah and M. R. Yadav, Recent advances in selective $\alpha_{1}$-adrenoreceptor antagonists as antihypertensive agents, Bioorg. Med. Chem., 2008, 16, 4759-4800.

22 J. Munín, E. Quezada, A. Cuiñas, M. Campos-Toimil, E. Uriarte, L. Santana and D. Viña, Synthesis, biological evaluation and structure-activity relationships of new phthalazinedione derivatives with vasorelaxant activity, Eur. J. Med. Chem., 2014, 82, 407-417.

23 S. Gatadi, T. V. Lakshmi and S. Nanduri, 4(3H)Quinazolinone derivatives: Promising antibacterial drug leads, Eur. J. Med. Chem., 2019, 170, 157-172.

24 D. Xie, J. Shi, A. Zhang, Z. Lei, G. Zu, Y. Fu, X. Gan, L. Yin, B. Song and D. Hu, Syntheses, antiviral activities and induced resistance mechanisms of novel quinazoline derivatives containing a dithioacetal moiety, Bioorg. Chem., 2018, 80, 433-443.

25 J. Zhang, J. Liu, Y. Ma, D. Ren, P. Cheng, J. Zhao, F. Zhang and Y. Yao, One-pot synthesis and antifungal activity against plant pathogens of quinazolinone derivatives containing an amide moiety, Bioorg. Med. Chem. Lett., 2016, 26, 2273-2277.

26 A. Bouchut, D. Rotili, C. Pierrot, S. Valente, S. Lafitte, J. Schultz, U. Hoglund, R. Mazzone, A. Lucidi, G. Fabrizi, D. Pechalrieu, P. B. Arimondo, T. S. Skinner-Adams, M. J. Chua, K. T. Andrews, A. Mai and J. Khalife, Identification of novel quinazoline derivatives as potent antiplasmodial agents, Eur. J. Med. Chem., 2019, 161, 277291.

27 K. P. Rakesh, H. M. Manukumar and D. C. Gowda, Schiff's bases of quinazolinone derivatives: synthesis and SAR studies of a novel series of potential anti-inflammatory and antioxidants, Bioorg. Med. Chem. Lett., 2015, 25, 1072-1077.

28 N. Sultana, M. Sarfraz, S. T. Tanoli, M. S. Akram, A. Sadiq, U. Rashid and M. I. Tariq, Synthesis, crystal structure determination, biological screening and docking studies of
$\mathrm{N}^{1}$-substituted derivatives of 2,3-dihydroquinazolin-4(1H)one as inhibitors of cholinesterases, Bioorg. Chem., 2017, 72, 256-267.

29 M. Sarfraz, N. Sultana, U. Rashid, M. S. Akram, A. Sadiq and M. I. Tariq, Synthesis, biological evaluation and docking studies of 2,3-dihydroquinazolin-4(1H)-one derivatives as inhibitors of cholinesterases, Bioorg. Chem., 2017, 70, 237244.

30 M. A. Qhobosheane, A. Petzer, J. P. Petzer and L. J. Legoabe, Synthesis and evaluation of 2-substituted 4(3H)quinazolinone thioether derivatives as monoamine oxidase inhibitors, Bioorg. Med. Chem., 2018, 26, 5531-5537.

31 G. Shi, X. He, Y. Shang, C. Yang and L. Xiang, Oxidative rearrangement of isatins with arylamines using $\mathrm{H}_{2} \mathrm{O}_{2}$ as oxidant: a facile synthesis of quinazoline-2,4-diones and evaluation of their antibacterial activity, Chin. J. Chem., 2017, 35, 1835-1843.

$32 \mathrm{~S}$. Petersen, H. Helmut and L. Born, Isatin-Ncarbonsäureamide und ihre Reaktionen, Justus Liebigs Ann. Chem., 1974, 12, 2003-2014.

33 L. Capuano and K. Benz, Spirocyclisierungen, Chem. Ber., 1977, 110, 3849-3861.

34 M. Yamagishi, K.- i. Ozaki, H. Ohmizu, Y. Yamada and M. Suzuki, Quinazolin-2-ones having a spirohydantoin ring. I. Synthesis of spiro[1,2,3,4-tetrahydroquinazoline4,4'-imidazolidine]-2,2',5'-trione by reaction of 1carbamoylisatin with urea or guanidine, Chem. Pharm. Bull., 1990, 38, 2926-2928.

35 S. Petersen, Dyestuffs and heterocycles inters- ie 3-substd 2keto-4-hydroxy-1,2,3,4-tetra hydro quinazoline-4-carboxylic acid hydrazides, Ger. Patent, DE2314242A1, 1973.

36 D. C. Palmer, e-EROS Encyclopedia of Reagents for Organic Synthesis, 2001, pp. 1-4.

37 A. F. Mabied, A. S. Girgis, E. M. Shalaby, R. F. George, B. E. M. El-Gendy and F. N. Baselious, Stereoselective synthesis, structural and spectroscopic study of 4,5,11triazatricyclo[6.2.1.0*2,6*] undec-5-ene, J. Heterocycl. Chem., 2016, 53, 1074-1080.

38 E. M. Shalaby, A. S. Girgis, H. Farag, A. F. Mabied and A. N. Fitch, Synthesis, X-ray powder diffraction and DFT calculations of vasorelaxant active 3-(arylmethylidene) pyrrolidine-2,5-diones, RSC Adv., 2016, 6, 112950-112959.

39 A. M. Srour, S. S. Panda, A. M. M. Salman, M. A. ElManawaty, R. F. George, E. M. Shalaby, A. N. Fitch, N. G. Fawzy and A. S. Girgis, Synthesis and molecular modeling studies of bronchodilatory active indole-pyridine conjugates, Future Med. Chem., 2018, 10, 1787-1804.

40 S. S. Panda, O. S. Detistov, A. S. Girgis, P. P. Mohapatra, A. Samir and A. R. Katritzky, Synthesis and molecular modeling of antimicrobial active fluoroquinolone-pyrazine conjugates with amino acid linkers, Bioorg. Med. Chem. Lett., 2016, 26, 2198-2205. 